\title{
High harmonics diffraction caused by an ellipticity grating
}

\author{
W Komatsubara ${ }^{1,2}$,, F Kong $^{1,3}$, C Zhang $^{1,3,4}$ and P B Corkum ${ }^{1,3}$ \\ ${ }^{1}$ Department of Physics, University of Ottawa, 25 Templeton St., Ottawa, ON K1N 6N5, Canada \\ ${ }^{2}$ Department of Physics, Graduate School of Science, The University of Tokyo, 7-3-1 Hongo, Bunkyo-ku, \\ Tokyo 113-0033, Japan \\ ${ }^{3}$ Joint Attosecond Science Laboratory, University of Ottawa and National Research Council of Canada 100 \\ Sussex Drive, Ottawa K1N 5A2, Canada
}

E-mail: Chunmei.Zhang@uottawa.ca

Received 17 December 2019, revised 2 February 2020

Accepted for publication 18 February 2020

Published 2 April 2020

\begin{abstract}
We study the generation of high-harmonic beams by the non-collinear wave mixing of two circularly or elliptically polarized fundamental beams. Changing the ellipticity of the two fundamental beams changes the electric field structure in the focal plane, generating multiple high harmonics beamlets. We show that the ellipticity dependence of the harmonic dipole modulates the near-field harmonic intensity, resulting in the diffraction of the generated harmonics in the far-field.
\end{abstract}

Keywords: high harmonics generation, diffraction, non-collinear-wave mixing, elliptical polarization

(Some figures may appear in colour only in the online journal)

\section{Introduction}

High-order harmonic generation (HHG) is a table-top laboratory light source at extreme ultraviolet wavelengths $[1,2]$. To create high harmonics, an electron in a gas [3-5], a solid [6-8] or a liquid [9] experiences an electric field from the laser similar to the Coulomb field.

The mechanism of HHG from gases is explained by the three-step model $[10,11]$ that shows that, as the polarization of the driving laser beam changes from linear to circular, the recombination probability and the harmonic yield both drop dramatically [3-5]. Therefore, high-harmonic experiments are generally performed with linearly polarized light, making linearly polarized harmonics. Initially it was not obvious how to generate the circularly-polarized harmonics that are in demand for such applications as probing chiral molecule [6-9, 12, 13], detecting magnetic circular dichroism [14-17].

However, studies have been conducted on generating circularly polarized high harmonics. Besides using a reflective quarter-wave plate (QWP) [18] or a symmetry-controlled media [19-21], one can control the polarization of the incident electric field in such a manner as to control the polarization of the high

\footnotetext{
4 Author to whom any correspondence should be addressed.
}

harmonics. For example one can use two-color counter-rotating circularly polarized beams [15-17, 22-26], non-collinear wave mixing [27-30] or vector beams [31]. We study non-collinear wave mixing.

Non-collinear wave mixing experiments can be explained by the momentum and spin angular momentum conservation $[32,33]$. This photon picture predicts the positions and ellipticities of the generated high-harmonic beams. In terms of the photon picture, the elliptically polarized beam is considered as the superposition of the right and left circularly polarized photons [33]. Linearly or circularly polarized incident beams have been mainly used in experiments studying conservation laws for high harmonics [24].

From a wave picture, non-collinear wave mixing forms a grating-like field modulation in the focal plane [27]. When the two incident beams are linearly polarized, the focused beams are also linearly polarized everywhere. The intensity distribution of the focused beams, however, forms an 'intensity grating', modulating the intensity distribution of the harmonics in the near-field and diffracting the multiple beamlets in the far-field. When it comes to circular polarization, in the focal plane the intensity distribution is Gaussian and the polarization is always linear with the polarization rotating as a function of the lateral position. This creates a 


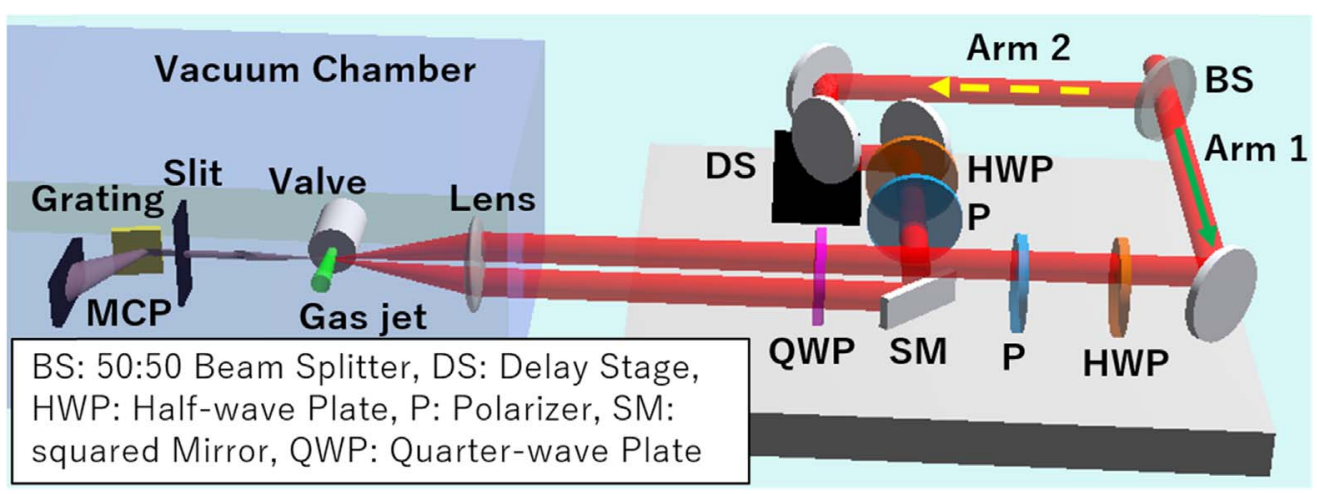

Figure 1. Experimental setup. The beam of Arm 1 goes above a squared mirror (SM) and that of Arm 2 is reflected.

'polarization-rotation grating' [27], resulting in the two beamlets in the far-field.

When the two incident beams are elliptically polarized, the intensity and polarization profiles might be similar to those of the circular polarization but the ellipticity oscillates forming an 'ellipticity grating' in addition to the 'polarization rotation grating'. The ellipticity grating creates the multiple beamlets in the far-field. In addition, an elliptically polarized incident beam modulates the electron trajectory in the continuum opening the potential to probe the dynamics of the electron wave packet and the symmetry of the molecular orbital [34-40].

In this paper, using a non-collinear geometry, we investigate high-harmonic generation by varying the ellipticities of the two fundamental beams. Changing the ellipticity of the incident beams modulate the ellipticity of the driving field in the focal plane, influencing the local high harmonic yield. The modulated harmonics are diffracted, creating multiple highharmonics beamlets. We use the wave picture to reveal the underlying physics of the high-harmonics diffraction caused by the ellipticity grating in the focus plane.

\section{Experiment}

The experimental setup is shown in figure 1. We use a Ti: sapphire laser system (Coherent Legend, pulse width 35 fs, central wavelength $780 \mathrm{~nm}$, pulse energy $2 \mathrm{~mJ}$ ). We split the beam with a 50:50 Beam Splitter (BS) into Arm 1 and Arm 2. In each optical path, the pulse energy and the angle of the linear polarization are adjusted by a half-wave plate (HWP) and a polarizer $(\mathrm{P})$. We adjust the polarization of the two beams to be orthogonal. The two beams are then combined by a squared mirror (SM). The Arm 1 beam goes above the SM and the Arm 2 beam is reflected so that the two beams spatially and temporally overlapped in the focal plane. The two beams then go through a QWP and the polarizations are converted from the orthogonally linear polarizations to the counter-rotating circular or elliptical polarizations. Two beams go into the vacuum chamber and are focused by a lens $(f=400 \mathrm{~mm})$ into a He gas jet. The generated high harmonics pass through the entrance slit of the spectrometer and are spectrally-separated by a grating, before being detected by a micro-channel plate (MCP). The florescent image of the MCP is collected by a camera.

\section{Results}

Figure 2 shows the polarization of the incident beams (a), (d) and the angles of the semi-major axis of the electric field ellipse (b), (e) and the ellipticities (c), (f). The angles and ellipticities are calculated using a two-dimensional Fourier transform based on the measured profiles of the beams. When the two incident beams are counter-rotating circularly polarized, the total electric field of the overlapped beams is linearly polarized, rotating several times, depending on the lateral separation between the two incident beams (figure 2(b)). For two counter-rotating elliptically polarized incident beams, the polarization angle (figure 2(e)) of the resultant field is almost the same as that shown in figure 2(b). However, the modulation of the ellipticity (figure 2(f)) is different from that shown in figure 2(c). When the circularly polarized beams are focused, the polarization in the focal plane is linear everywhere (figure 2(c)), but the ellipticity oscillates between about -0.04 and 0.04 when the elliptically polarized incident beams are focused (figure 2(f)). This slight ellipticity modulation makes a big difference; the diffraction of the generated high harmonics as discussed below.

Figure 3(a) shows two high-harmonic beamlets generated by the counter-rotating circularly polarized incident beams. We fit the two beamlets to a Gaussian function and plot their center positions in figure 3(b). The positions are consistent with the theoretical lines also plotted in figure 3(b) (see Discussion section equation (6)).

Compared to the high harmonics generated by the circularly-polarized beams (figure 3(a)), the high harmonics from the elliptically polarized beams have their first order at the same angle and also produces many more orders of diffracted radiation in the vertical direction (figure 3(c)). We fit the beamlets to a Gaussian function and plot their center positions in figure 3(d). The zero position in the vertical direction is defined by the center of the +1 and -1 diffraction orders. The experimental results are consistent with the theoretical positions of the beamlets written by the solid lines in figure 6(d). (see Discussion section equation (6)).

We also measure the polarization of the two beamlets in figure 3(a) and verify that the two beamlets are counterrotating circularly polarized [27]. 

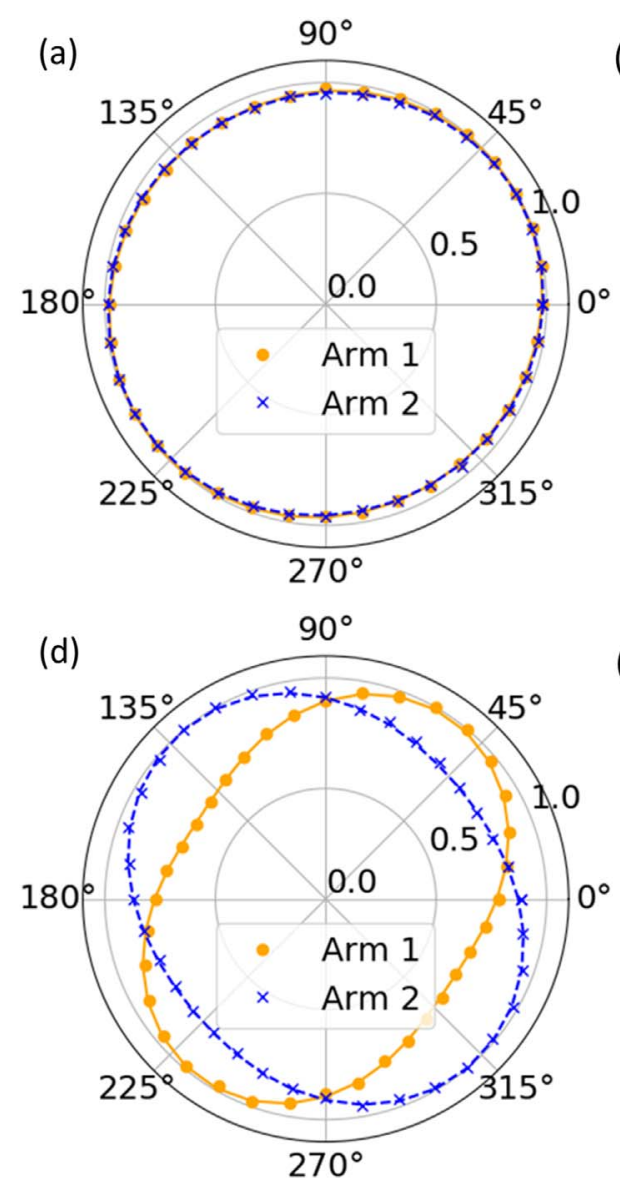

(b)

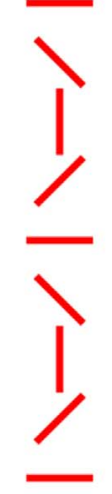

(e)

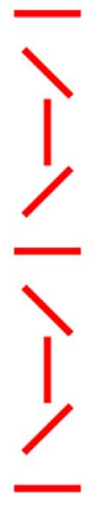

(c)

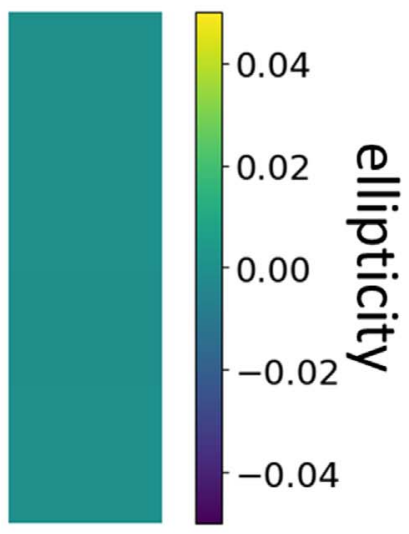

(f)

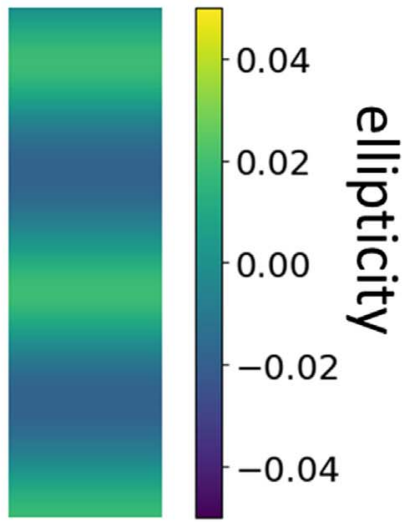

Figure 2. Experimental results of polarization and ellipticity of the two fundamental beams before and after focusing. (a) Polarization of the circularly polarized incident beams. Each intensity is normalized. The ellipticity of both Arm 1 and 2 are 0.97 . (b) The angle of the semimajor axis of the electric field ellipse. (c) The ellipticity in the focal plane. (d) Polarization of the elliptically polarized incident beams. Each intensity is normalized. The ellipticity of Arm 1 is 0.83 and that of Arm 2 is 0.89 . (e) The angle of the semi-major axis of the electric field ellipse. (f) The ellipticity in the focal plane.

In order to measure the polarization of the high harmonics, a HWP is inserted before the lens and a polarizer consisted of two Au mirror is introduced between the valve and the slit. For each pixel of the image data, the ellipticity is calculated based on the HWP's angle dependence of the high harmonic intensity. It should be noted that since we can measure only the amplitude of the ellipticity with this method, the range of the ellipticity is from 0 to 1 . Figure 4 shows the profiles of the intensity (a) and the ellipticity (b) of the 15th order harmonic in figure 3(a). The ellipticity near the peaks of the two beamlets is close to 1 , which means the polarization of the two beamlets is circular, while the ellipticity in the region between the two beamlets is close to 0 , indicating the polarization is linear. These two results suggest that the two beamlets are counter-rotating circularly polarized.

\section{Discussion}

Here we examine the mechanism of the high-harmonic generation in terms of the wave picture [27]. We work in the normalized space for the $q$ th order harmonic in figure 5, where both focusing of the incident beams by the lens and the propagation of the $q$ th order harmonic are expressed by the two dimensional Fourier transform. (We return to this property at the end of the Discussion section.)

First, however, we discuss why multiple beamlets are diffracted by the elliptically polarized incident beams in terms of the wave picture (figure 5(b)). The derivation in detail is given in appendix B. As shown in figures 2 (c) and (f), the difference between the focused circularly and elliptically polarized incident beams is the ellipticity modulation in the focal plane, which is written as

$$
\varepsilon \approx \varepsilon_{\text {const }} \sin 2 t^{\prime} y_{2}^{\prime}
$$

with $\varepsilon_{\text {const }}$ is a constant, $\left(x_{2}^{\prime}, y_{2}^{\prime}\right)$ are the coordinates of the normalized space for the $q$ th order harmonic in the focal plane. This ellipticity oscillation modulates the intensity of the harmonic in the near-field. The harmonic intensity $I(\varepsilon)$ depends on the ellipticity $\varepsilon$ expressed as [3-5]

$$
I(\varepsilon) \propto \exp \left(-\frac{\varepsilon^{2}}{\varepsilon_{t h}^{2}}\right)
$$

with $\varepsilon_{t h}$ the threshold ellipticity dependent on the intensity of the driving beam, the harmonic order, the wavelength, and the ionization potential of the medium. The electric field of the 


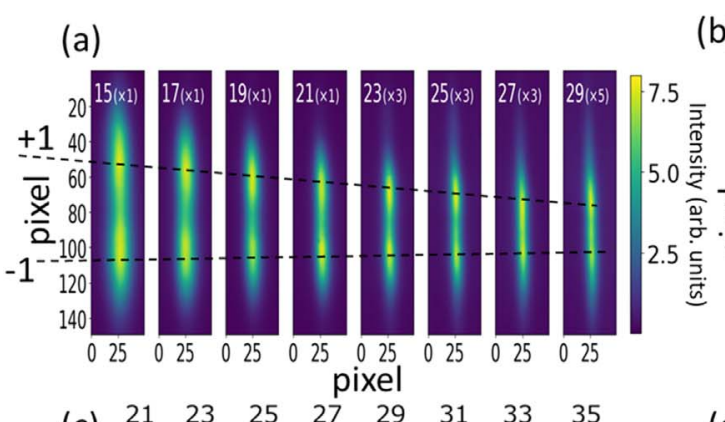

(b)
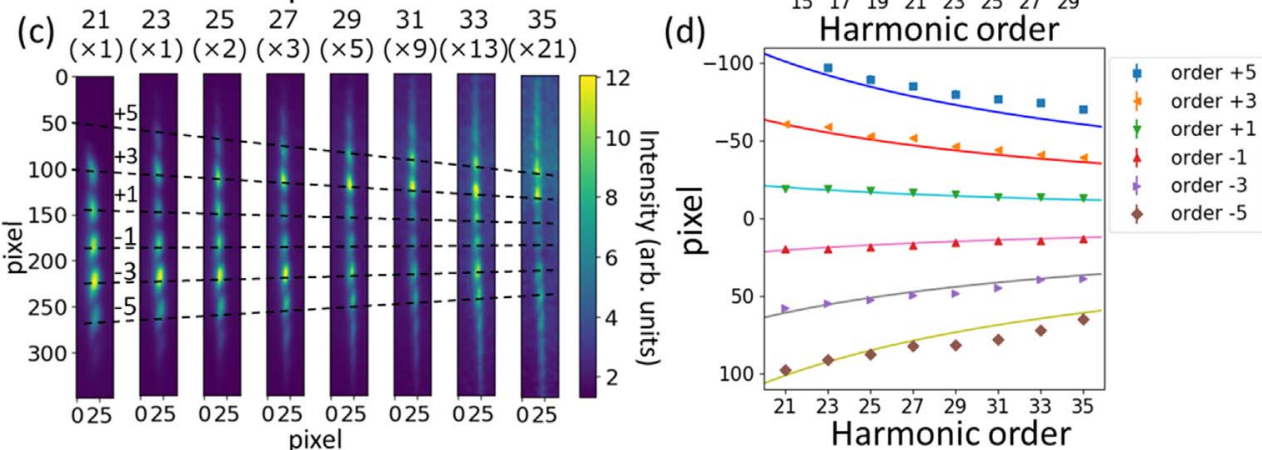

Figure 3. High harmonics images. (a) High harmonics generated by the counter-rotating circularly-polarized incident beams. The numbers under the harmonic orders show how much the intensities are enhanced compared to the actual intensities. The beamlets in (a) are fitted by a Gaussian function and their center positions are plotted in (b). Numbers of $-1,+1$ are the diffraction orders. (c) High harmonics generated by the counterrotating elliptically-polarized incident beams. The dashed lines in (c) represent each beamlet of the diffracted high harmonics. The beamlets in (c) are fitted by a Gaussian function and their center positions are plotted in (d). Numbers of $-5,-3,-1,+1,+3,+5$ are the diffraction orders.

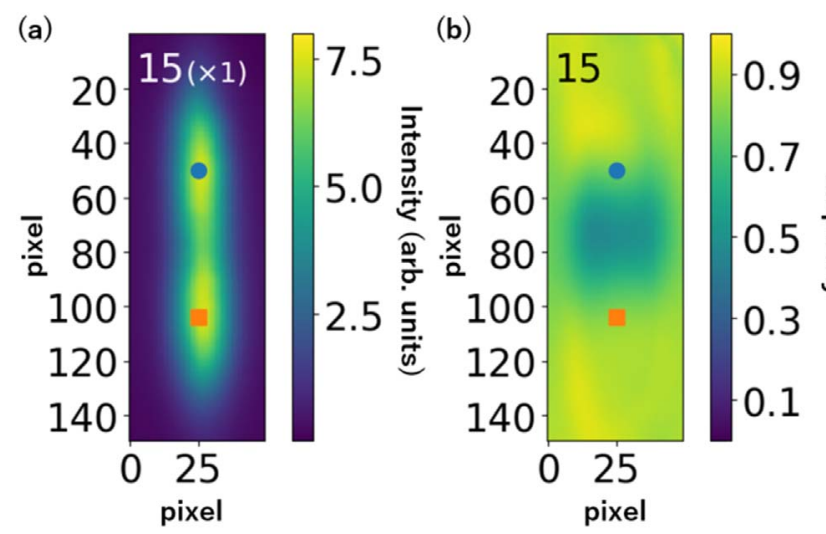

Figure 4. Profiles of intensity (a) and ellipticity (b) of the 15th order harmonic in figure 3 (a). The circle and square markers plotted in (a) and (b) show the peak positions of the two beamlets.

$q$ th order harmonic in the near-field can be written as

$$
\begin{aligned}
& \underset{E_{\text {near }, q}}{\longrightarrow}\left(x_{2}^{\prime}, y_{2}^{\prime}\right) \approx \exp \left(-\frac{x_{2}^{\prime 2}+y_{2}^{\prime 2}}{4}\right)\left(\begin{array}{c}
\cos t^{\prime} y_{2}^{\prime} \\
-\sin t^{\prime} y_{2}^{\prime}
\end{array}\right) \\
& \times \exp \left(-\frac{1}{2} \frac{\varepsilon_{\text {const }}^{2} \sin ^{2} 2 t^{\prime} y_{2}^{\prime}}{\varepsilon_{t h}^{2}}\right) .
\end{aligned}
$$

Here, the terms $\left(\begin{array}{c}\cos t^{\prime} y_{2}^{\prime} \\ -\sin t^{\prime} y_{2}^{\prime}\end{array}\right)$ and $\exp \left(-\frac{1}{2} \frac{\varepsilon_{\text {const }}^{2} \sin ^{2} 2 t^{\prime} y_{2}^{\prime}}{\varepsilon_{t h}^{2}}\right)$ represent the polarization rotation (see appendix C) and the amplitude modulation, respectively. The electric field of the

\section{(a) Circular polarized beams}

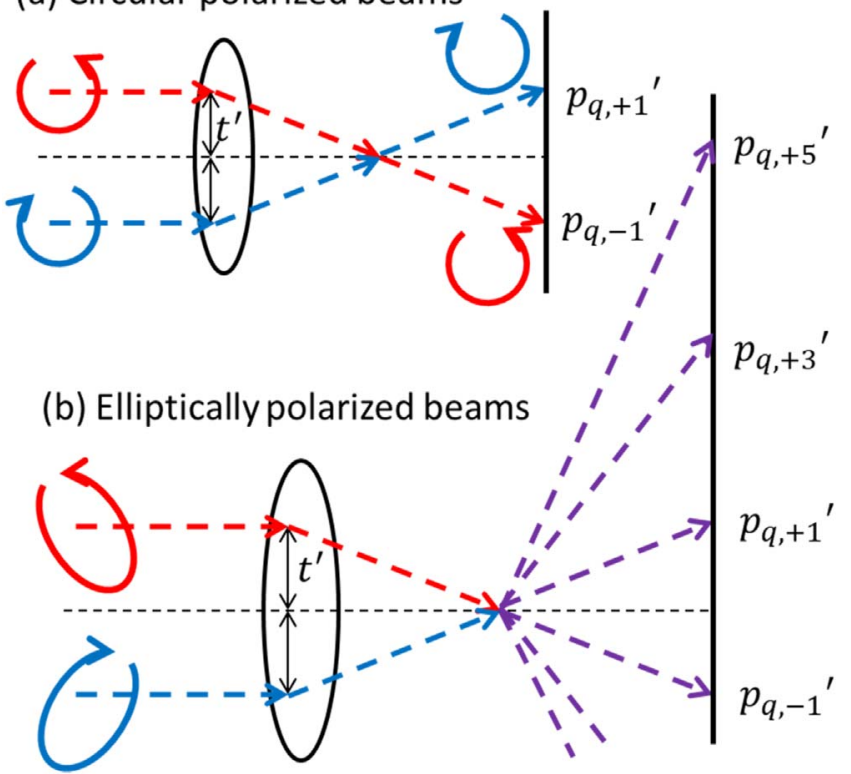

Figure 5. Normalized space for the $q$ th order harmonic. The separation between the two incident beams is defined as $2 t^{\prime}$. (a) When the two incident beams are circularly polarized, two beamlets are diffracted. The corresponding positions of the two beamlets are expressed as $p_{q, \pm 1}{ }^{\prime}$. The indices \pm 1 represent the diffraction orders of the two harmonic beamlets. (b) When the two incident beams are elliptically polarized, the generated harmonics are diffracted. The corresponding positions of the diffracted beamlets are expressed as $p_{q, \pm 1}{ }^{\prime}, p_{q, \pm 3}{ }^{\prime}, p_{q, \pm 5}{ }^{\prime} \cdots$. The indices $\pm 1, \pm 3, \pm 5$ represent the diffraction orders of the beamlets. The detail definition of the normalized space for the $q$ th order harmonic is written in appendix B. 


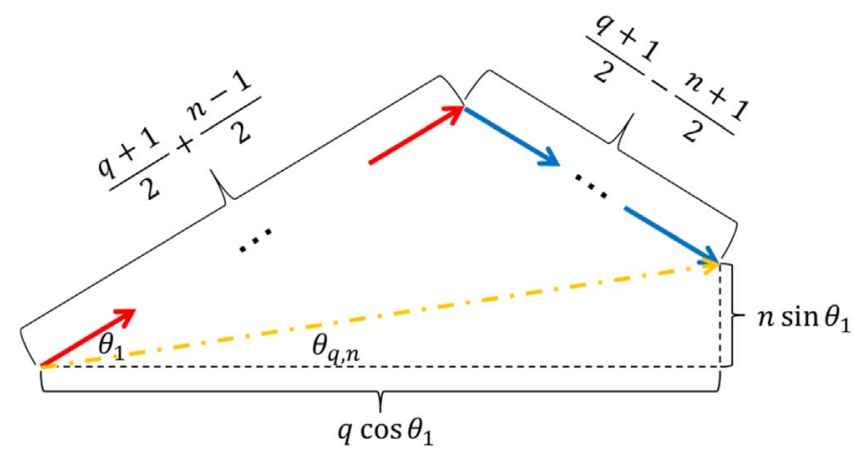

Figure 6. Beamlets positions based on the photon picture.

$q$ th order harmonic in the far-field can be then expressed as

$$
\begin{aligned}
& \vec{E}_{f a r, q}\left(x_{3, q}^{\prime}, y_{3, q}^{\prime}\right) \propto \iint \overrightarrow{E_{\text {near }, q}}\left(x_{2}^{\prime}, y_{2}^{\prime}\right) \\
& \quad \times \exp \left\{-i\left(x_{2}^{\prime} x_{3, q}^{\prime}+y_{2}^{\prime} y_{3, q}^{\prime}\right)\right\} d x_{2}^{\prime} d y_{2}^{\prime} \\
& \quad=\sum_{k=1} C_{2 k-1}\left[\left(\begin{array}{l}
1 \\
i
\end{array}\right) \exp \left(-x_{3, q}^{\prime 2}-\left(y_{3, q}^{\prime}-(2 k-1) t^{\prime}\right)^{2}\right)\right. \\
& \left.+\left(\begin{array}{c}
1 \\
-i
\end{array}\right) \exp \left(-x_{3, q}^{\prime 2}-\left(y_{3, q}^{\prime}+(2 k-1)^{\prime}\right)^{2}\right)\right]
\end{aligned}
$$

with $\left(x_{3, q}^{\prime}, y_{3, q}^{\prime}\right)$ the coordinates of the normalized space for the $q$ th order harmonic in the far-field. The $C_{1}$ terms $(k=1)$ in equation (4) correspond to the diffracted beamlets $p_{q, \pm 1}{ }^{\prime}$ located at $\pm t^{\prime}$. In the same way, the $C_{3}$ terms $(k=2)$ correspond to the diffracted beamlets $p_{q, \pm 3}{ }^{\prime}$ located at $\pm 3 t^{\prime}$ and the $C_{5}(k=3)$ terms the diffracted beamlets $p_{q, \pm 5}{ }^{\prime}$ located at $\pm 5 t^{\prime}$. It should be noted that each beamlet is actually elliptically polarized, but due to the approximations, equation (4) shows that each beamlet is circularly polarized. Therefore, the positions of the beamlets $p_{q, \pm n}^{\prime}$ can be written as

$$
p_{q, \pm n}^{\prime} \approx \pm n t^{\prime}
$$

In the experimental space, equation (5) can be interpreted in terms of the angle of the incident and $q$ th harmonic beams as follows: (see appendix B (B27))

$$
\tan \theta_{q, \pm n}= \pm \frac{n}{q} \tan \theta
$$

Equation (6) can be also derived based on the spin and momentum conservation laws of the photon.

The beamlets position $p_{q, n}^{\prime}$ in the case of $n>0$ is calculated based on the momentum conservation law as shown in figure 6 . The $n$th beamlet of the $q$ th order harmonic absorbs $\left(\frac{q+1}{2}+\frac{n-1}{2}\right)$ incident photons corresponding to the upward arrows and $\left(\frac{q+1}{2}-\frac{n+1}{2}\right)$ incident photons corresponding to the downward arrows, resulting in

$$
\tan \theta_{q, n}=\frac{n \sin \theta_{1}}{q \cos \theta_{1}}=\frac{n \tan \theta_{1}}{q} .
$$

Here we consider the elliptically polarized beam as including both right- and left-handed circularly polarized photons [33]. We also examine the validity of equation (5) (see appendix C.2).

Next, we think about the two circularly polarized incident beams, which are the special case of the elliptically polarized beams. The reason why the circularly polarized beams generate only two beamlets in the far-field is because of the lack of the ellipticity oscillation. When the two incident beams are circularly polarized, the focused beams are linearly polarized everywhere, i.e. $\varepsilon=0$ in the focal plane. This makes the amplitude modulation term $\exp \left(-\frac{1}{2} \frac{\varepsilon_{\text {const }}^{2} \sin ^{2} 2 t^{\prime} y_{2}{ }^{\prime}}{\varepsilon_{t h}^{2}}\right)$ always 1 in equation (3), resulting in no beamlets corresponding to $C_{3}, C_{5}, \cdots$ except for $C_{1}$ in equation (4). Therefore, there are only two beamlets with counter-rotating circular polarization in the far-field when the two incident beams are circularly polarized.

We can understand the reason why the circularly polarized beams generate only two beamlets in the far-field differently. As mentioned above, in this normalized space, focusing of the incident beams by the lens and the propagation of the $q$ th order harmonic are expressed as the same twodimensional Fourier transform as follows:

$$
\begin{aligned}
& \overrightarrow{E_{f}}\left(x_{2}^{\prime}, y_{2}^{\prime}\right) \propto \iint \overrightarrow{E_{i n}}\left(x_{1}^{\prime}, y_{1}^{\prime}\right) \\
& \quad \times \exp \left\{-i\left(x_{1}^{\prime} x_{2}^{\prime}+y_{1}^{\prime} y_{2}^{\prime}\right)\right\} d x_{1}^{\prime} d y_{1}^{\prime},
\end{aligned}
$$

and

$$
\begin{gathered}
\underset{E_{f a r}, q}{\longrightarrow}\left(x_{3, q}^{\prime}, \quad y_{3, q}^{\prime}\right) \propto \iint \overrightarrow{E_{\text {near }, q}}\left(x_{2}^{\prime}, y_{2}^{\prime}\right) \\
\quad \times \exp \left\{-i\left(x_{2}^{\prime} x_{3, q}^{\prime}+y_{2}^{\prime} y_{3, q}^{\prime}\right)\right\} d x_{2}^{\prime} d y_{2}^{\prime},
\end{gathered}
$$

where $\overrightarrow{E_{f}}\left(x_{2}^{\prime}, y_{2}^{\prime}\right)$ is the electric field in the focal plane, $\overrightarrow{E_{\text {in }}}\left(x_{1}^{\prime}, y_{1}^{\prime}\right)$ is the incident electric field, $\overrightarrow{E_{f a r}, q}\left(x_{3, q}^{\prime}, y_{3, q}^{\prime}\right)$ and $\overrightarrow{E_{\text {near, } q}}\left(x_{2}^{\prime}, y_{2}^{\prime}\right)$ is the qth order harmonic electric field in the far- and near-field, respectively, and $\left(x_{1}^{\prime}, y_{1}^{\prime}\right)$ are the coordinates of the lens of the normalized space for the qth order harmonic. In this space, the two circularly polarized incident beams are written as

$$
\begin{aligned}
& \overrightarrow{E_{\text {in }}}\left(x_{1}^{\prime}, y_{1}^{\prime}\right) \propto\left(\begin{array}{c}
1 \\
-i
\end{array}\right) \exp \left(-x_{1}^{\prime 2}-\left(y_{1}^{\prime}-t^{\prime}\right)^{2}\right)+\left(\begin{array}{l}
1 \\
i
\end{array}\right) \\
& \quad \times \exp \left(-x_{1}^{\prime 2}-\left(y_{1}^{\prime}+t^{\prime}\right)^{2}\right) .
\end{aligned}
$$

Therefore, focusing of the incident beams by the lens and the propagation of the $q$ th order harmonic are expressed as the same two-dimensional Fourier transform in equations (8) and (9). Therefore, the incident beams $\overrightarrow{E_{i n}}\left(x_{1}^{\prime}, y_{1}^{\prime}\right)$ should be the same as the $q$ th harmonic in the far field $\overrightarrow{E_{f a r}, q}\left(x_{3, q}^{\prime}, y_{3, q}^{\prime}\right)$, which indicates that the position of the incident beam $t^{\prime}$ and that of the $q$ th harmonic $p_{q, \pm 1}^{\prime}$ are also the same:

$$
p_{q, \pm 1}^{\prime} \approx \pm t^{\prime}
$$


This relationship can be also derived using spin and momentum conservation laws of the photon (see equation (7)). We examine the validity of equation (11) with numerical calculations (see appendix C.1).

\section{Conclusion}

Typically, experiments studying the ellipticity dependence of high-harmonic generation have used spatially uniform polarization beams. In contrast we show that focused beams with ellipticity leads to a modulated high harmonics intensity in the near-field. The diffracted high harmonics then propagate to form multiple beamlets in the far-field. These multiple beamlets are generated at the same place in the far-field as when the incident beams are linearly polarized. When the incident beams are circularly polarized, the diffracted beamlets vanish. Two circularly polarized fundamental beams create only a polarization rotation grating, generating only two beamlets in the far-field. In contrast, the two elliptically polarized beams form both a polarization rotation grating and an ellipticity grating in the focal plane. The ellipticity grating enforces multiple diffracted beamlets in the far-field.

The ellipticity dependence of the harmonic intensity has many applications [34-40]. Elliptically polarized beams modulate the electron trajectory in the continuum [34], helpful for probing the dynamics of the electron wave packet. This manipulation of the electron trajectory has an influence on the recombination probability and will be applicable to probing the property of the molecular orbitals, especially in chiral systems [8].

Since the intensities of the beamlets depend on the threshold ellipticity $\varepsilon_{t h}$ in equation (2), which is relevant to the medium, the ellipticity dependence of each diffracted beamlet will provide new insight into the medium. Although the diffracted beamlets of the diffraction orders \pm 1 are generated by both linearly and elliptically/circularly polarized incident beams, the other diffracted beamlets vanish when the incident beams are circularly polarized, which means the background free measurement of the ellipticity dependence. We believe that elliptically polarized beams can combine the ellipticity dependence experiments to create a new 'ellipticity grating spectroscopy' [41].

\section{Acknowledgments}

The authors are pleased to acknowledge the support of Dr Chunmei Zhang, Yu-Hsuan Wang from University of Ottawa throughout this work. We also acknowledge important financial support from the US DARPA Topological Excitations in Electronics (TEE) program, grant D18AC00011. In addition, we acknowledge the support of the Canadian Canada Research Chairs; the Natural Sciences and Engineering Research Council of Canada; the Canadian Foundation for Innovation programs. $\mathrm{W} \mathrm{K}$ is grateful for the financial support from the Advanced Leading Graduate Course for Photon Science (ALPS) founded by the Ministry of Education, Culture, Sports, Science, and Technology (MEXT). This work was (partially) supported by JST COI Grant Number JPMJCE1313.
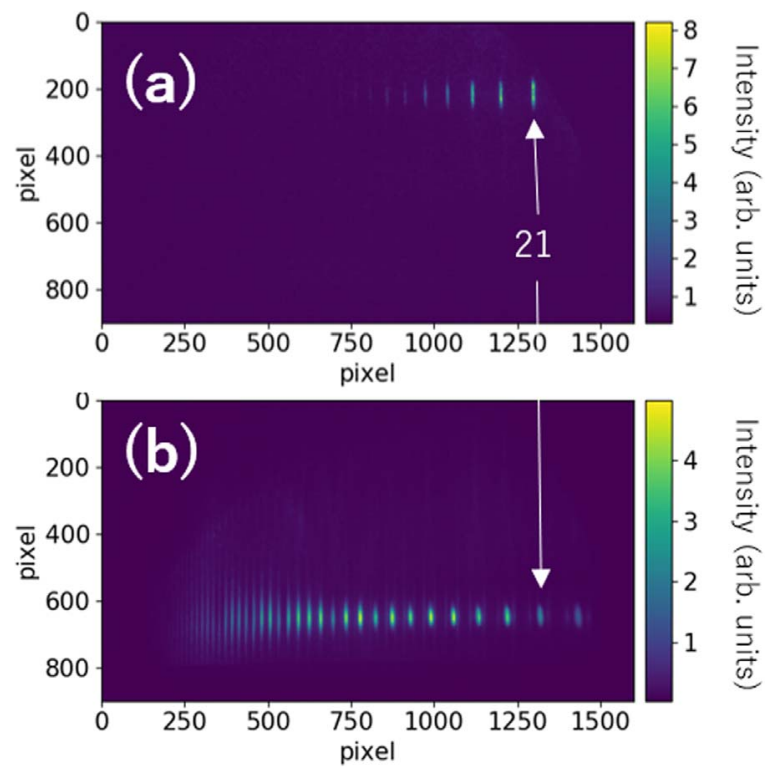

Figure A1. High harmonics spectra generated in (a) the noncollinear geometry by the two counter-rotating circularly polarized fundamental beams and (b) by the linearly polarized fundamental beam of the Arm 1. The number ' 21 ' in figure A1 means the harmonic order.

\section{Appendix A.}

\section{Efficiency of HHG in the noncollinear configuration}

To investigate the efficiency of HHG, we compare the high harmonics generated in the noncollinear geometry by the two counter-rotating circularly polarized fundamental beams (a) with those generated by the linearly polarized fundamental beam of the Arm 1 (b) (the fundamental beam of the Arm 2 is blocked). The total pulse energies of the incident beams are the same ((a) Arm 1: $0.5 \mathrm{~mJ}$, Arm 2: $0.5 \mathrm{~mJ}$, (b) Arm 1: $1 \mathrm{~mJ}$ ). In spite of the same total pulse energy, the cutoffs of the high harmonics are different: about 40 for (a) and about 90 for (b). From these cutoffs, the effective intensities of the focal point are estimated at (a) $2.0 \times 10^{14} \mathrm{~W} \mathrm{~cm}^{-2}$ and (b) $5.2 \times 10^{14} \mathrm{~W} \mathrm{~cm}^{-2}$. The estimated effective intensity for (a) is lower than that for (b) because of the difference in the configurations and the polarizations of the incident beams. Therefore, it is suggested that the efficiency of HHG in the noncollinear geometry is about one third lower than that in the general experiment with using the single linearly polarized fundamental beams in terms of the effective intensity of the focal point.

\section{Appendix B. Derivation of the beamlets positions when the incident beams are counter-rotating elliptically polarized}

The elliptically polarized beam is generally written as

$$
E_{x}=a_{1} e^{i \theta}, E_{y}=a_{2}
$$

with $a_{1}, a_{2}$ and $\theta_{1}$ real numbers. Since here we consider the elliptically polarized beams as almost the circularly polarized 


\section{Experimental space}
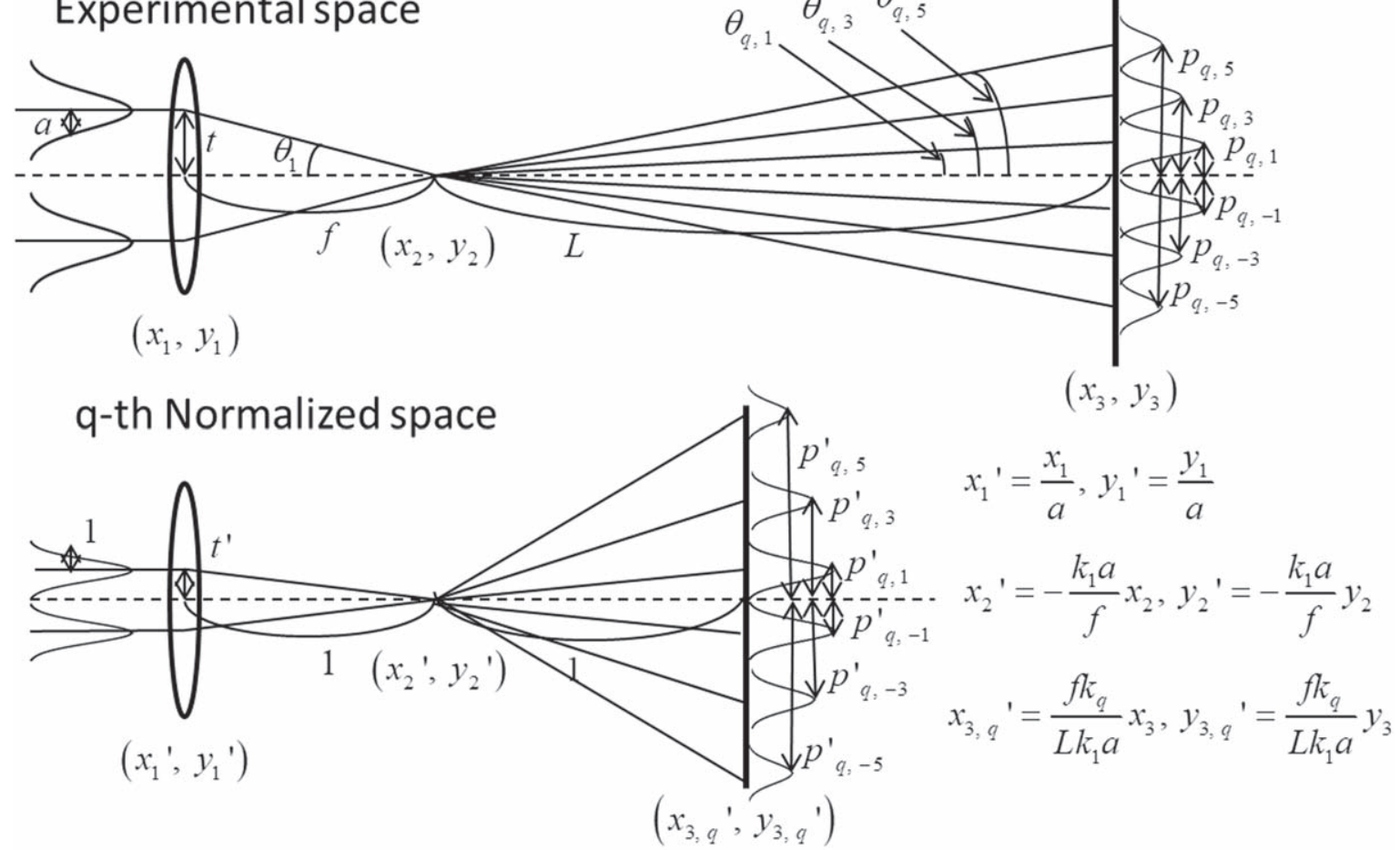

Figure B1. Relationship between the coordinate of the experimental space and that of the normalized space for the $q$ th order harmonic.

ones, we can assume

$$
\theta \approx \frac{\pi}{2} \text { and } a_{1} \approx \pm a_{2}
$$

Next, we define the normalized space for the $q$ th order harmonic in figure B1. In this space, focusing of the incident beams by the lens and the propagation of the $q$ th order harmonic are expressed by the same two-dimensional Fourier transform of equations (8) and (9). The coordinate of the lens $\left(x_{1}, y_{1}\right)$ in the experimental space is normalized such

$$
x_{1}^{\prime}=\frac{x_{1}}{a} \text { and } y_{1}^{\prime}=\frac{y_{1}}{a}
$$

with $a$, the incident beams' radius. The coordinate of the focal plane $\left(x_{2}, y_{2}\right)$ in the experimental space is normalized such that

$$
x_{2}^{\prime}=-\frac{k_{1} a}{f} x_{2} \text { and } y_{2}^{\prime}=-\frac{k_{1} a}{f} y_{2}
$$

with $k_{1}$ the wave vector of the incident beams and $f$ the focal length. The coordinate of the far field $\left(x_{3, q}, y_{3, q}\right)$ in the experimental space is normalized such that

$$
x_{3, q^{\prime}}=\frac{f k_{q}}{L k_{1} a} x_{3} \text { and } y_{3, q^{\prime}}=\frac{f k_{q}}{L k_{1} a} y_{3}
$$

with $k_{q}$ the wave vector of the $q$ th order harmonic and $L$ the distance between the focal point and the screen in the far field.
The non-collinear counter-rotating elliptically polarized incident beams are expressed by

$$
\begin{aligned}
\overrightarrow{E_{\text {in }}} & =\left(\begin{array}{c}
E_{1 x} e^{i \theta_{1}} \\
E_{1 y}
\end{array}\right) \exp \left(-x_{1}{ }^{2}-\left(y_{1}^{\prime}-t^{\prime}\right)^{2}\right) \\
& +\left(\begin{array}{c}
E_{2 x} e^{i \theta_{2}} \\
-E_{2 y}
\end{array}\right) \exp \left(-x_{1}{ }^{\prime 2}-\left(y_{1}^{\prime}+t^{\prime}\right)^{2}\right),
\end{aligned}
$$

where $t^{\prime}\left(=\frac{t}{a}\right)$ is the normalized positions of the two incident beams as shown in figure B1. $E_{1 x}, E_{1 y}, E_{2 x}$ and $E_{2 y}$ are the amplitudes of the two incident beams $\left(E_{1 x}, E_{1 y}, E_{2 x}, E_{2 y}>0\right)$ and $\theta_{1}, \theta_{2}$ are the phases, respectively. In this experiment, $E_{1 x}: E_{1 y}=1: 1.08, \theta_{1}=1.40, E_{2 x}: E_{2 y}=1: 1.02$ and $\theta_{2}=$ 1.69, which is consistent with the assumption of equation (B2). The focused beams can be obtained by two dimensional Fourier transform as follows:

$$
\begin{aligned}
\overrightarrow{E_{f}} & \propto \exp \left(-\frac{x_{2}^{\prime 2}+y_{2}^{\prime 2}}{4}\right) \times\left(\left(\begin{array}{c}
E_{1 x} e^{i \theta_{1}} \\
E_{1 y}
\end{array}\right) \exp \left(-i t^{\prime} y_{2}^{\prime}\right)\right. \\
& \left.+\left(\begin{array}{c}
E_{2 x} e^{i \theta_{2}} \\
-E_{2 y}
\end{array}\right) \exp \left(i t^{\prime} y_{2}^{\prime}\right)\right) .
\end{aligned}
$$

Here, we substitute $\theta_{1} \approx \frac{\pi}{2}, \theta_{2} \approx \frac{\pi}{2}$ based on equation (B2) into (B7) and get

$$
\begin{aligned}
& \overrightarrow{E_{f}} \approx \exp \left(-\frac{x_{2}^{\prime 2}+y_{2}^{\prime 2}}{4}\right) \\
& \times\left(\begin{array}{l}
\left(E_{1 x}-E_{2 x}\right) \sin t^{\prime} y_{2}^{\prime}+i\left(E_{1 x}+E_{2 x}\right) \cos t^{\prime} y_{2}^{\prime} \\
\left(E_{1 y}-E_{2 y}\right) \cos t^{\prime} y_{2}^{\prime}-i\left(E_{1 y}+E_{2 y}\right) \sin t^{\prime} y_{2}^{\prime}
\end{array}\right) .
\end{aligned}
$$


By calculating the Stokes parameter, we can obtain the ellipticity

$$
\varepsilon=\tan \alpha,
$$

where

$$
\sin 2 \alpha=\frac{2 b_{1} b_{2} \sin \left(\varphi_{1}-\varphi_{2}\right)}{b_{1}^{2}+b_{2}^{2}}
$$

Here, $b_{1}, b_{2}, \varphi_{1}, \varphi_{2}$ are given by

$$
\begin{gathered}
\overrightarrow{E_{f}}=\exp \left(-\frac{x_{2}^{\prime 2}+y_{2}^{\prime 2}}{4}\right) \times\left(\begin{array}{l}
b_{1} e^{i \varphi_{1}} \\
b_{2} e^{i \varphi_{2}}
\end{array}\right), \\
b_{1}=\sqrt{E_{1 x^{2}+E_{2 x}{ }^{2}+2 E_{1 x} E_{2 x} \cos 2^{\prime} y_{2}{ }^{\prime}}}, \\
b_{2}=\sqrt{E_{1 y^{2}+E_{2 y}{ }^{2}-2 E_{1 y} E_{2 y} \cos 2 t^{\prime} y_{2}{ }^{\prime}},} \\
\sin \varphi_{1}=\frac{\left(E_{1 x}+E_{2 x}\right) \cos t^{\prime} y_{2}{ }^{\prime}}{b_{1}}, \text { and } \\
\sin \varphi_{2}=\frac{-\left(E_{1 y}+E_{2 y}\right) \sin t^{\prime} y_{2}{ }^{\prime}}{b_{2}} .
\end{gathered}
$$

Now the elliptically polarized beams are almost circularly polarized. That is:

$$
E_{1 x} \approx E_{1 y} \approx E_{2 x} \approx E_{2 y} \approx E .
$$
we get

We substitute equations (B12), (B13), (B16) into (B10) and

$$
\sin 2 \alpha \approx \frac{2 \sqrt{2 E^{2}\left(1+\cos 2 t^{\prime} y_{2}^{\prime}\right)} \sqrt{2 E^{2}\left(1-\cos 2 t^{\prime} y_{2}^{\prime}\right)} \sin \left(\varphi_{1}-\varphi_{2}\right)}{4 E^{2}}=\sin 2 t^{\prime} y_{2}^{\prime} \sin \left(\varphi_{1}-\varphi_{2}\right)
$$

Based on equations (B9) and (B17), we obtain

$$
\varepsilon=\tan \left(\frac{1}{2} \arcsin \left(\sin 2 t^{\prime} y_{2}^{\prime} \sin \left(\varphi_{1}-\varphi_{2}\right)\right)\right)
$$

Here, we substitute equation (B16) into (B14) and (B15) and get

$$
\varphi_{1} \approx \frac{\pi}{2}, \varphi_{2} \approx-\frac{\pi}{2}
$$

and we can assume

$$
\sin 2 t^{\prime} y_{2}^{\prime} \sin \left(\varphi_{1}-\varphi_{2}\right) \ll 1
$$

Therefore, we obtain

$$
\begin{aligned}
\varepsilon= & \tan \left(\frac{1}{2} \arcsin \left(\sin 2 t^{\prime} y_{2}{ }^{\prime} \sin \left(\varphi_{1}-\varphi_{2}\right)\right)\right) \\
& \approx \tan \left(\frac{1}{2} \sin 2 t^{\prime} y_{2}^{\prime} \sin \left(\varphi_{1}-\varphi_{2}\right)\right) \\
& \approx \frac{1}{2} \sin 2 t^{\prime} y_{2}^{\prime} \sin \left(\varphi_{1}-\varphi_{2}\right) .
\end{aligned}
$$

In summary, the period of the ellipticity modulation can be calculated as

$$
\varepsilon=\varepsilon_{\mathrm{const}} \sin 2 t^{\prime} y_{2}^{\prime}
$$

with $\varepsilon_{\text {const }}$ a constant dependent on the incident beams (see equation (B21)). Here, because the ellipticity modulation is very weak (see figure 2(f) and equation (B21)), we assume that the ellipticity oscillation have an influence only on the harmonic intensity, not the polarization. In other words, we can approximate the polarization of the near-field $q$ th order harmonic $\overrightarrow{E_{\text {near }, q}}\left(x_{2}^{\prime}, y_{2}^{\prime}\right)$ as linear, and $\overrightarrow{E_{\text {near }, q}}\left(x_{2}^{\prime}, y_{2}^{\prime}\right)$ is proportional to the product of the focused counter-rotating circularly polarized beams

$$
\overrightarrow{E_{\text {in, circular }}}\left(x_{2}^{\prime}, y_{2}^{\prime}\right) \propto \exp \left(-\frac{x_{2}^{\prime 2}+y_{2}^{\prime 2}}{4}\right)\left(\begin{array}{c}
\cos t^{\prime} y_{2}^{\prime} \\
-\sin t^{\prime} y_{2}^{\prime}
\end{array}\right)
$$

while the amplitude modulation term equation (2) as follows:

$$
\begin{aligned}
& \underset{E_{\text {near }, q}}{\longrightarrow}\left(x_{2}^{\prime}, y_{2}^{\prime}\right) \approx \exp \left(-\frac{x_{2}^{\prime 2}+y_{2}^{\prime 2}}{4}\right)\left(\begin{array}{c}
\cos t^{\prime} y_{2}^{\prime} \\
-\sin t^{\prime} y_{2}^{\prime}
\end{array}\right) \\
& \times \exp \left(-\frac{1}{2} \frac{\varepsilon_{\text {const }}^{2} \sin ^{2} 2 t^{\prime} y_{2}^{\prime}}{\varepsilon_{t h}^{2}}\right) .
\end{aligned}
$$

It should be noted that in equation (B24) we approximate the amplitude profile of the harmonics by the focused incident beams to simplify the equation. This approximation is validated by numerical calculation of appendix D.2. Equation (B23) is derived from equation (B8) by using equation (B16). The electric fields of the high harmonics in the far-field can be then written as:

$$
\begin{aligned}
& \underset{E_{f a r}, q}{\longrightarrow}\left(x_{3, q}^{\prime}, y_{3, q}^{\prime}\right) \propto \iint \exp \left(-\frac{x_{2}^{\prime 2}+y_{2}^{\prime 2}}{4}\right) \\
& \times\left(\begin{array}{c}
\cos t^{\prime} y_{2}^{\prime} \\
-\sin t^{\prime} y_{2}^{\prime}
\end{array}\right) \exp \left(-\frac{\varepsilon_{\text {const }}^{2} \sin ^{2} 2 t^{\prime} y_{2}^{\prime}}{2 \varepsilon_{\text {th }}^{2}}\right) \\
& \times \exp \left\{-i\left(x_{2}^{\prime} x_{3, q}^{\prime}+y_{2}^{\prime} y_{3, q}^{\prime}\right)\right\} d x_{2}^{\prime} d y_{2}^{\prime} \\
& =\iint \exp \left(-\frac{x_{2}^{\prime 2}+y_{2}^{\prime 2}}{4}\right)\left\{C_{1}\left(\begin{array}{c}
\cos t^{\prime} y_{2}^{\prime} \\
-\sin t^{\prime} y_{2}^{\prime}
\end{array}\right)\right. \\
& \left.+C_{3}\left(\begin{array}{c}
\cos 33^{\prime} y_{2}^{\prime} \\
-\sin 3 t^{\prime} y_{2}^{\prime}
\end{array}\right)+C_{5}\left(\begin{array}{c}
\cos 5 t^{\prime} y_{2}^{\prime} \\
-\sin 5 t^{\prime} y_{2}^{\prime}
\end{array}\right)+\cdots\right\} \\
& \times \exp \left\{-i\left(x_{2}^{\prime} x_{3, q}^{\prime}+y_{2}^{\prime} y_{3, q}^{\prime}\right)\right\} d x_{2}^{\prime} d y_{2}^{\prime} \\
& \propto C_{1}\left[\left(\begin{array}{l}
1 \\
i
\end{array}\right) \exp \left(-x_{3, q}^{\prime 2}-\left(y_{3, q}^{\prime}-t^{\prime}\right)^{2}\right)\right.
\end{aligned}
$$




$$
\begin{aligned}
& \left.+\left(\begin{array}{c}
1 \\
-i
\end{array}\right) \exp \left(-x_{3, q}^{\prime 2}-\left(y_{3, q}^{\prime}+t^{\prime}\right)^{2}\right)\right] \\
& +C_{3}\left[\left(\begin{array}{l}
1 \\
i
\end{array}\right) \exp \left(-x_{3, q}^{\prime 2}-\left(y_{3, q}^{\prime}-3 t^{\prime}\right)^{2}\right)\right. \\
& \left.+\left(\begin{array}{c}
1 \\
-i
\end{array}\right) \exp \left(-x_{3, q}^{\prime 2}-\left(y_{3, q}^{\prime}+3 t^{\prime}\right)^{2}\right)\right] \\
& +C_{5}\left[\left(\begin{array}{l}
1 \\
i
\end{array}\right) \exp \left(-x_{3, q}^{\prime 2}-\left(y_{3, q}^{\prime}-5 t^{\prime}\right)^{2}\right)\right. \\
& \left.+\left(\begin{array}{c}
1 \\
-i
\end{array}\right) \exp \left(-x_{3, q}^{\prime 2}-\left(y_{3, q}^{\prime}+5 t^{\prime}\right)^{2}\right)\right]+\cdots
\end{aligned}
$$

The $C_{1}$ terms in equation (B25) correspond to the diffracted beamlets $p_{q, \pm 1}{ }^{\prime}$ located at $\pm t^{\prime}$. In the same way, the $C_{3}$ terms correspond to the diffracted beamlets $p_{q, \pm 3}{ }^{\prime}$ located at $\pm 3 t^{\prime}$ and the $C_{5}$ terms the diffracted beamlets $p_{q, \pm 5}{ }^{\prime}$ located at $\pm 5 t^{\prime}$. Each beamlet is actually elliptically polarized, but due to the approximations, equation (B25) shows that each beamlet is circularly polarized.

Equation (B25) shows that the positions of the beamlets $p_{q, \pm n}^{\prime}$, written as

$$
p_{q, \pm n}^{\prime} \approx \pm n t^{\prime}
$$

yields

$$
\begin{aligned}
p_{q, \pm n} & =\frac{a L}{q f} p_{q, \pm n}^{\prime} \approx \pm \frac{a L}{q f} n t^{\prime}= \pm \frac{L t}{q f} n \\
& = \pm n \frac{L}{q} \tan \theta_{1}=L \tan \theta_{q, \pm n} .
\end{aligned}
$$

Equation (B27) is consistent with the photon picture of equation (B7).

In equation (B25) we have used the following relationships:

$$
\begin{aligned}
& \cos x \exp \left(-a \sin ^{2} 2 x\right)=c_{1} \cos x+c_{3} \cos 3 x \\
& \quad+c_{5} \cos 5 x+\cdots, \text { and } \\
& \sin x \exp \left(-a \sin ^{2} 2 x\right)=c_{1} \sin x+c_{3} \sin 3 x \\
& \quad+c_{5} \sin 5 x+\cdots .
\end{aligned}
$$

Equations (B28) and (B29) can be derived as follows:

$$
\begin{aligned}
& e^{i x} \exp \left(-a \sin ^{2} x\right) \\
& =e^{i x} \sum_{n=0}^{\infty} \frac{(-a)^{n}}{n !}(\sin x)^{2 n} \\
& =e^{i x} \sum_{n=0}^{\infty} \frac{(-a)^{n}}{n !}\left(\frac{e^{i x}-e^{-i x}}{2 i}\right)^{2 n} \\
& =e^{i x} \sum_{n=0}^{\infty} \frac{(-a)^{n}}{n !} \sum_{k=0}^{2 n}\left(\begin{array}{c}
2 n \\
k
\end{array}\right)\left(\frac{1}{2 i}\right)^{2 n}\left(e^{i x}\right)^{k}\left(e^{-i x}\right)^{2 n-k} \\
& =\sum_{n=0}^{\infty} \frac{(-a)^{n}}{n !}\left(\frac{-1}{4}\right)^{n} \sum_{k=0}^{2 n}\left(\begin{array}{c}
2 n \\
k
\end{array}\right)\left(e^{i x}\right)^{2(k-n)+1} .
\end{aligned}
$$

The real and imaginary part of equation (B30) show equations (B28) and (B29), respectively.

\section{Appendix C. Numerical calculation}

We calculate the high harmonics based on the Lewenstein model [42].

\section{C.1. Counter-rotating circularly polarized incident beams}

The focused incident beams are expressed by equation (B23). The peak intensity of the focused beams is $1.0 \times 10^{14} \mathrm{~W} \mathrm{~cm}^{-2}$. The near-field electric field of the $q$ th order harmonic is then written as

$$
\underset{E_{\text {near }, q}}{\longrightarrow}\left(x_{2}^{\prime}, y_{2}^{\prime}\right) \approx E_{H H G, q}\left(x_{2}^{\prime}, y_{2}^{\prime}\right)\left(\begin{array}{c}
\cos t^{\prime} y_{2}^{\prime} \\
-\sin t^{\prime} y_{2}^{\prime}
\end{array}\right),
$$

where $E_{H H G, q}\left(x_{2}^{\prime}, y_{2}^{\prime}\right)$ is the calculated amplitude of the $q$ th order harmonic. It should be noted that in order to simplify the problem, we do not consider the effect of phase-matching [43, 44] and we assume that the phase of the near-field harmonic electric field $\overrightarrow{E_{n e a r}, q}$ is the same as that of the focused incident beams $\overrightarrow{E_{\text {in,circular }}}$ as shown in equation (C1). The far-field electric field of the $q$ th order harmonic is calculated according to equation (9).
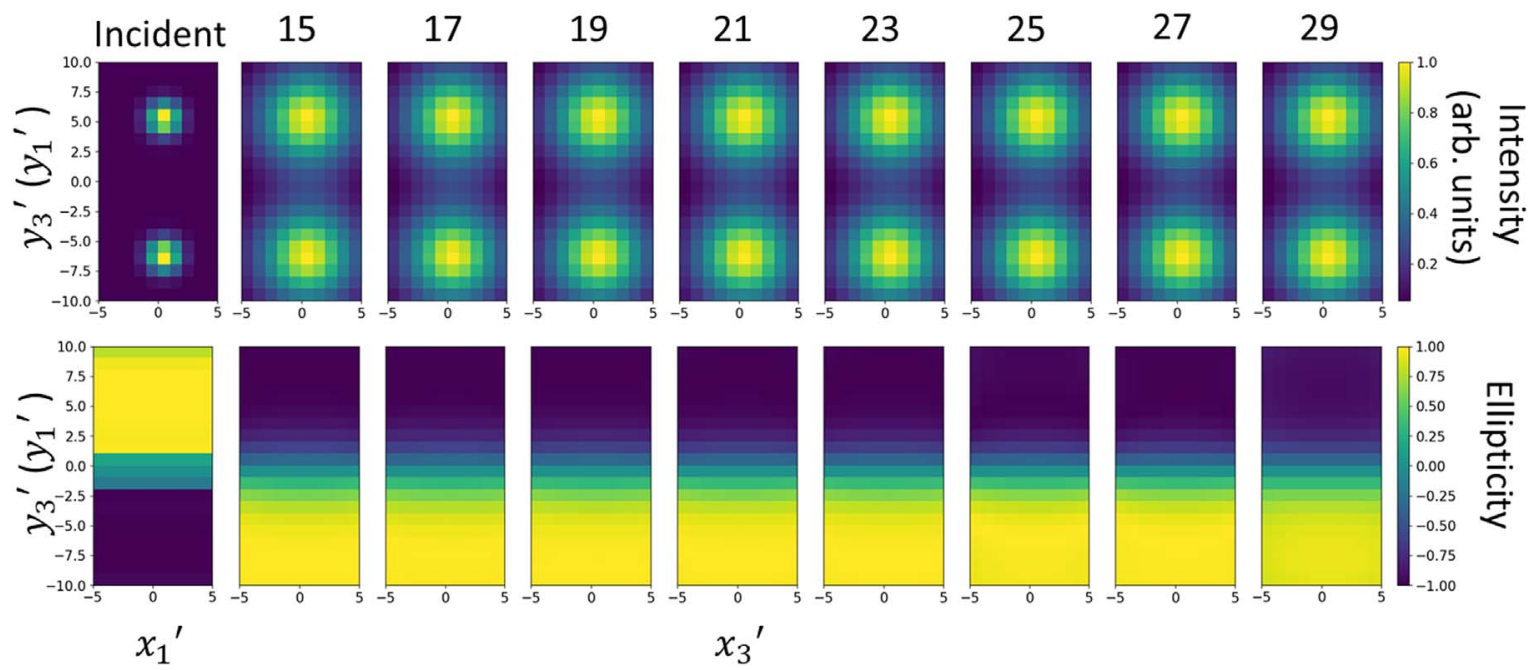

Figure C1. Numerical calculation results of the high harmonics generated by the counter-rotating circularly polarized incident beams. The left column shows the intensity and ellipticity profile of the incident beams and the others those of from the 15th to 29th order harmonics. 


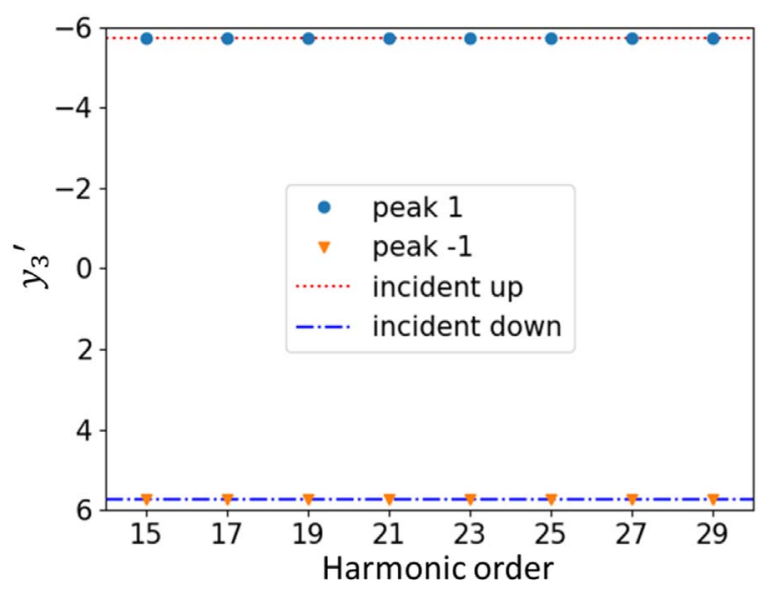

Figure C2. Peak positions of the two beamlets.
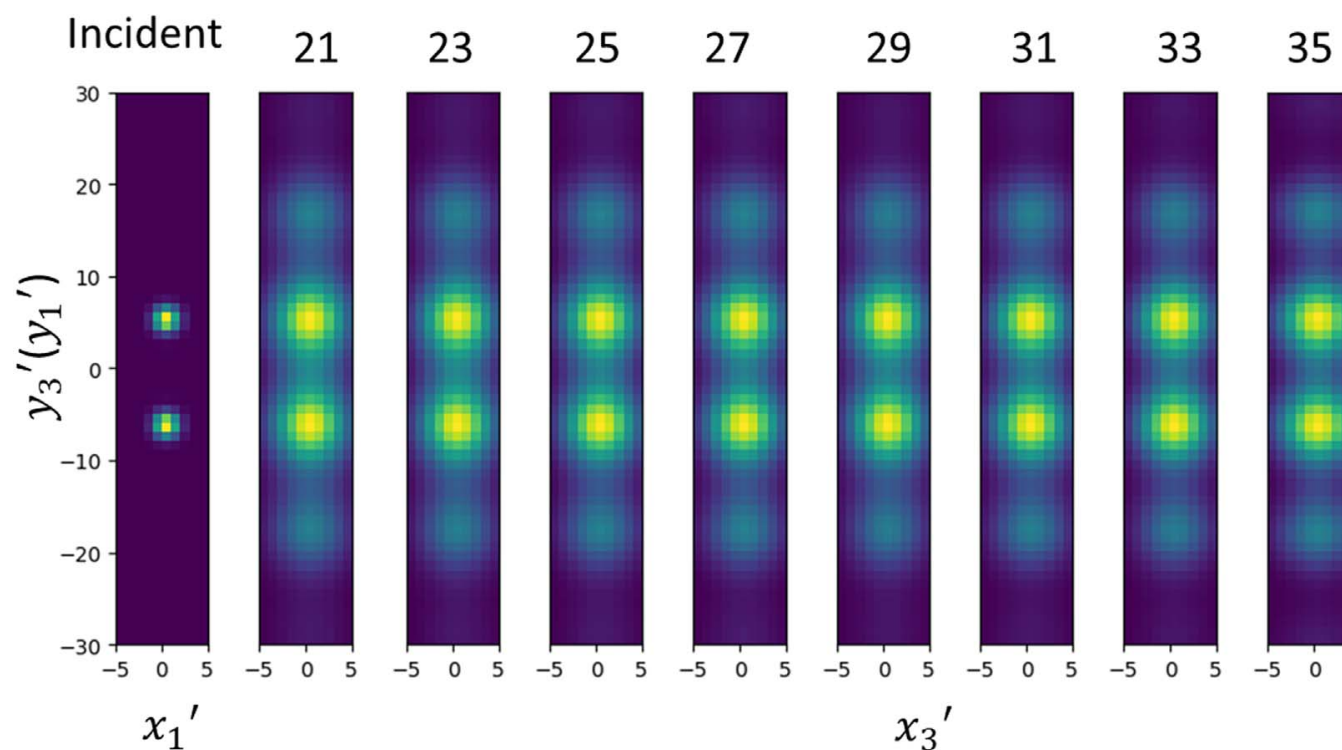

Figure C3. Numerical calculation results of the high harmonics generated by the counter-rotating elliptically polarized incident beams. The left column shows the intensity profile of the incident beams and the others that of from the 21 st to 35 th order harmonics.

Since we pay attention to the peak positions of the high harmonics, we normalize each intensity of the incident beams and high harmonics. Figure $\mathrm{C} 1$ shows that the peak positions of the upper and lower incident beams are almost the same as those of the two beamlets of the high harmonics, respectively. Also, the ellipticity profiles in figure $\mathrm{C} 1$ show that the ellipticities of the two beamlets are counter-rotating circular polarization.

We fit the intensity profiles in figure $\mathrm{C} 1$ by a Gaussian function and the peak positions are plotted in figure $\mathrm{C} 2$. The two dashed lines show the peak positions of the upper and lower incident beams, respectively. As shown in figure $\mathrm{C} 2$, the peak positions of the two beamlets are independent of the harmonic order and consistent with those of the two incident beams, which supports the conclusion of equation (11).

\section{C.2. Counter-rotating elliptically polarized incident beams}

The focused incident beams are expressed by equation (B7). The peak intensity of the focused beams is $1.3 \times 10^{14} \mathrm{~W} \mathrm{~cm}{ }^{-2}$. The near-field electric field of the $q$ th order harmonic is written as

$$
\begin{aligned}
& \underset{E_{\text {near }, q}}{\longrightarrow}\left(x_{2}^{\prime}, y_{2}^{\prime}\right) \approx\left(\begin{array}{c}
\cos t^{\prime} y_{2}^{\prime} \\
-\sin t^{\prime} y_{2}^{\prime}
\end{array}\right) E_{H H G, q}\left(x_{2}^{\prime}, y_{2}^{\prime}\right) \\
& \times \exp \left(-\frac{1}{2} \frac{\varepsilon_{\text {const }}^{2} \sin ^{2} 2 t^{\prime} y_{2}^{\prime}}{\varepsilon_{\text {th }}^{2}}\right) .
\end{aligned}
$$

The difference between equation (B24) and equation (C2) is the amplitude term. In the former case, we approximate the amplitude profile of the harmonics by using the focused incident beams. Here, we use the calculated amplitude of the harmonic $E_{H H G, q}\left(x_{2}^{\prime}, y_{2}^{\prime}\right)$ generated by the linearly polarized beams. Though the polarization of the focused beams is not precisely linear, the ellipticity modulation is very small, which enables us to consider the polarization of the high harmonics as linear. The amplitude modulation is expressed by the term equation (2). Each threshold ellipticity in equation (2) is calculated for each order harmonic based on [5]. The propagation is calculated in the same way as appendix C.1. 


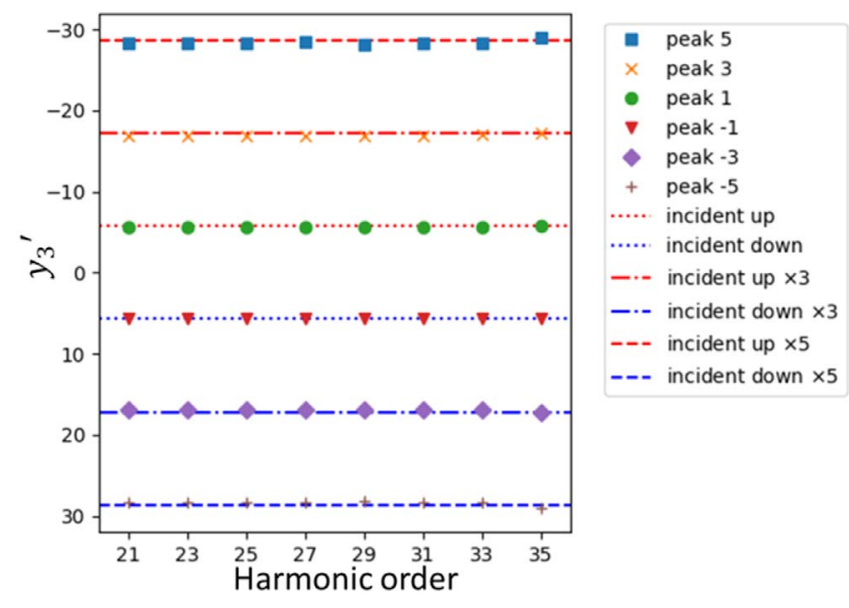

Figure C4. Peak positions of the diffracted beamlets.

We normalize each intensity of the incident beams and high harmonics. Figure $\mathrm{C} 3$ shows the amplitude modulation expressed in equation (B24) diffracts the harmonic beamlets.

We fit the intensity profiles in figure $\mathrm{C} 1$ by a Gaussian function and the peak positions are plotted in figure $\mathrm{C} 2$. The six dashed lines show the peak positions of the upper and lower incident beams $\pm t^{\prime}$, and their odd multiple positions $\pm 3 t^{\prime}, \pm 5 t^{\prime}$, respectively. As shown in figure $\mathrm{C} 4$, the peak positions of the diffracted beamlets are independent of the harmonic order and almost the same as those of the incident beams and their odd multiple ones, which supports the conclusion of equation (5).

\section{ORCID iDs}

W Komatsubara (ib https://orcid.org/0000-0003-2846-3317

\section{References}

[1] Corkum P B and Krausz F 2007 Nat. Phys. 3381

[2] Krausz F and Ivanov M 2009 Rev. Mod. Phys. 81163

[3] Möller M, Cheng Y, Khan S D, Zhao B, Zhao K, Chini M, Paulus G G and Chang Z 2012 Phys. Rev. A 86 011401(R)

[4] Khan S D, Cheng Y, Möller M, Zhao K, Zhao B, Chini M, Paulus G G and Chang Z 2011 Appl. Phys. Lett. 99161106

[5] Strelkov V V, Khokhlova M A, Gonoskov A A, Gonoskov I A and Ryabikin M Y 2012 Phys. Rev. A 86 013404

[6] Harada Y, Haraguchi E, Kaneshima K and Sekikawa T 2018 Phys. Rev. A 981

[7] Liu X, Lan P, Zhu X, Lu P, Li W, Zhang Q and Wang D 2016 Opt. Express 2424824

[8] Cireasa R et al 2015 Nat. Phys. 11654

[9] Ferré A et al 2015 Nat. Photon. 993
[10] Corkum P B 1993 Phys. Rev. Lett. 711994

[11] Schafer K J, Yang B, Dimauro L F and Kulander K C 1993 Phys. Rev. Lett. 701599

[12] Beaulieu A et al 2018 Nat. Phys. 14484

[13] Baykusheva D and Wörner H J 2018 Phys. Rev. X 8031060

[14] Boeglin C, Beaurepaire E, Halté V, López-Flores V, Stamm C, Pontius N, Dürr H A and Bigot J Y 2010 Nature 465458

[15] Kfir O et al 2015 Nat. Photon. 999

[16] Legut D et al 2015 Proc. Natl Acad. Sci. 11214206

[17] Lambert G et al 2015 Nat. Commun. 66167

[18] Lambert G et al 2011 Opt. Express 194346

[19] Houzet J, Hertz E, Billard F, Lavorel B and Faucher O 2013 Phys. Rev. A 881

[20] Skantzakis E, Chatziathanasiou S, Carpeggiani P A, Sansone G, Nayak A, Gray D, Tzallas P, Charalambidis D, Hertz E and Faucher O 2016 Sci. Rep. 61

[21] Xia P, Itatani J, Lu F, Kanai T, Saito N and Ishii N 2017 Optica 41333

[22] Eichmann H, Egbert A, Nolte S, Momma C, Wellegehausen B, Becker W, Long S and McIver J K 1995 Phys. Rev. A 51 R3414

[23] Milošević D B, Becker W and Kopold R 2000 Phys. Rev. A 6115

[24] Cohen O, Diskin T, Kfir O, Fleischer A and Sidorenko P 2014 Nat. Photon. 8543

[25] Kfir O, Bordo E, Ilan Haham G, Lahav O, Fleischer A and Cohen O 2016 Appl. Phys. Lett. 108211106

[26] Dorney K M et al 2017 Phys. Rev. Lett. 11945

[27] Hickstein D D et al 2015 Nat. Photon. 9743

[28] Hernández-García C et al 2016 Phys. Rev. A 93043855

[29] Grychtol P et al 2017 Opt. Express 2510126

[30] Huang P C et al 2018 Nat. Photon. 12349

[31] Kong F et al 2019 Nat. Commun. 101

[32] Bertrand J B et al 2011 Phys. Rev. Lett. 106023001

[33] Pisanty E, Sukiasyan S and Ivanov M 2014 Phys. Rev. A 901

[34] Komatsubara W, Minemoto S and Sakai H 2018 Phys. Rev. A 981

[35] Shan B, Ghimire S and Chang Z 2004 Phys. Rev. A 69 021404(R)

[36] Flettner A, König J, Mason M B, Pfeifer T, Weichmann U and Gerber G 2003 J. Mod. Opt. 50529

[37] Mairesse Y, Dudovich N, Levesque J, Ivanov M Y, Corkum P B and Villeneuve D M 2008 New J. Phys. 10 025015

[38] Larsen E W, Carlström S, Lorek E, Heyl C M, Paleček D, Schafer K J, L'Huillier A, Zigmantas D and Mauritsson J 2016 Sci. Rep. 639006

[39] Flettner A, König J, Mason M B, Pfeifer T, Weichmann U, Düren R and Gerber G. 2002 Eur. Phys. J. D 21115

[40] Kanai T, Minemoto S and Sakai H 2007 Phys. Rev. Lett. 98 053002

[41] Mairesse Y, Zeidler D, Dudovich N, Spanner M, Levesque J, Villeneuve D M and Corkum P B 2008 Phys. Rev. Lett. 10015

[42] Lewenstein M, Balcou P, Ivanov M Y, L'Huillier A and Corkum P B 1994 Phys. Rev. A 492117

[43] Rego L, Román J S, Picón A, Plaja L and Hernández-García C 2016 Phys. Rev. Lett. 1171

[44] Hernández-García C, Pérez-Hernández J A, Ramos J, Jarque E C, Roso L and Plaja L 2010 Phys. Rev. A 821 